\title{
Fixed point theorems in uniformly rotund metric spaces
}

\section{John Staples}

In recent years fixed point theorems have been proved for nonexpansive and similar mappings on uniformly convex Banach spaces. The only role the linear structure plays in the statement of these results occurs in the definition of uniform convexity. It is therefore natural to ask whether the results depend essentially on the linear structure, or whether an extension of the notion of uniform convexity to metric spaces would allow the hypothesis of linear structure on the underlying space to be removed.

In this paper such an extension is given; however the extended concept is called uniform rotundity since uniformly rotund metric spaces need not be convex. The use of uniform rotundity allows the hypothesis of linear structure to be removed from fixed point theorems of Edelstein, and Cooper and Michael. A consequence is that the original results are strengthened, so as to apply to a wider class of functions on uniformly convex Banach spaces.

\section{Introduction}

The link between uniform rotundity and fixed points of functions is a suitable notion of centre of a set or sequence. The functions being considered are used to define a suitable set or sequence, uniform rotundity allows the existence of a unique centre to be proved, and it follows that this centre is a fixed point. In what follows we consider two types of centre.

Received 12 November 1975. The author thanks Dr H. Bevan Thompson for helpful criticism of a draft of this paper.

181 
The following notation will be used. $M$ denotes a metric space, $d$ its metric, $S$ a non-empty bounded subset of $M$, and for any $c \in M$ and any nonnegative real number $r, B(c, r)$ denotes

$$
\{x \in M: d(c, x) \leq r\} \text {. }
$$

Similarly, $B(S, \boldsymbol{r})$ denotes

$$
\{x \in M \text { : for some } s \in S, d(s, x) \leq r\} \text {. }
$$

A set $B(c, r)$ as above may be called a ball, with centre $c$ and radius $r$.

1.1. If there is a ball $B(c, r)$ of minimum radius which contains $S$, then $c$ is called a Chebychev centre of $S$ in $M$, and $r$ is called the Chebychev radius of $S$ in $M$. The Chebychev radius can be defined more generally, to be the greatest lower bound of numbers $r$ such that for some $c \in M, S \subseteq B(c, r)$.

1.2. Consider a bounded sequence $\left(x_{n}\right)$ of points of $M$. The greatest lower bound of numbers $r$ such that for some $c \in M$,

$$
\lim \sup \left\{d\left(x_{n}, c\right)\right\} \leq r \text {, }
$$

is called the asymptotic radius of $\left(x_{n}\right)$. An asymptotic centre of $\left(x_{n}\right)$ is any $c \in M$ which minimizes $\lim \sup \left\{d\left(x_{n}, c\right)\right\}$. These two notions are due to Edelstein [2]. For example, a Cauchy sequence is just a sequence with asymptotic radius zero, and its limit, if any, is its only asymptotic centre.

\section{Uniqueness of Chebychev and asymptotic centres}

A very weak notion of uniform rotundity, as follows, is sufficient to prove uniqueness of Chebychev centres. We continue the notation of the previous section.

2.1. A set $N$ is called locally uniformly rotund in $M$, if for all $x, y \in M, x \neq y$, and all positive real numbers $\rho$, there is $z \in M$ such that

$$
N \cap B(x, \rho) \cap B(y, \rho) \subseteq B(z, r),
$$

for some real number $r<\rho$. 
In any case there will be a greatest lower bound $m$ of such numbers $r$; the difference $\rho-m$ may be denoted $\delta_{N}(x, y, z, \rho)$. The condition that $N$ is locally uniformly rotund in $M$ may then be stated as follows: for all $x, y \in M, x \neq y$, and all real $\rho>0$, there is $z \in M$ such that

$$
\delta_{N}(x, y, z, \rho)>0
$$

If $M$ is locally uniformly rotund in $M$, we say simply that $M$ is locally uniformly rotund.

2.2. The following uniqueness result extends Cooper and Michael [ 1 , Theorem 2.4].

If $S \subseteq M$ is locally zoniformly rotund in $M$, then $S$ has at most one Chebychev centre in $M$.

Proof. If $S$ has two distinct Chebychev centres $x$ and $y$, then $S$ has positive Chebycher radius in $M$, say $\rho$, so (2.I) there is $z \in M$ such that

$$
S=S \cap B(x, \rho) \cap B(y, \rho) \subseteq B(z, r)
$$

for some $r<\rho$, contradicting the definition of the Chebychev radius $\rho$.

2.3. It is convenient for applications of 2.2 to note that:

If $N \subseteq M$ is nonempty and $M$ is locally uniformly rotund, then $N$ is locally uniformly rotund in $M$.

2.4. To obtain unique asymptotic centres, local uniform rotundity needs strengthening as follows: a nonempty set $N$ is called regularly locally uniformly convex in $M$ if, in the notation of 2.1, for all $x, y \in M$ and all real positive $p$ and $q, p<q$, there is $z \in M$ such that

$$
\rho_{N}(x, y, z, p, q)=\inf _{p \leq \rho \leq q} \delta_{N}(x, y, z, \rho)>0
$$

In particular, if $M$ is regularly locally uniformly convex in $M$, then $M$ is called regularly locally uniformly convex.

2.5. If $M$ is regularly locatly zoniformly convex and $\left(x_{n}\right)$ is a sequence of elements of $M$, then $\left(x_{n}\right)$ has at most one asymptotic centre. 
Proof. Suppose on the contrary that $\left(x_{n}\right)$ has two distinct asymptotic centres $x$ and $y$; then $\left(x_{n}\right)$ is bounded, say $x_{n} \in B(x, k) \cap B(y, k)$ for all $n$, and the asymptotic radius $\rho$ of $\left(x_{n}\right)$ is positive. Thus, in the notation of 2.4 , for some $z \in M$ and all but finitely many $n$,

$$
\begin{aligned}
& d\left(x, x_{n}\right) \leq \rho+\frac{1}{2} \cdot \rho_{M}(x, y, z, \rho, \rho+k), \\
& d\left(y, x_{n}\right) \leq \rho+\frac{1}{2} \cdot \rho_{M}(x, y, z, \rho, \rho+k),
\end{aligned}
$$

where $\rho_{M}(x, y, z, \rho, \rho+k)$ is positive, and so for all but finitely many $n$,

$$
\begin{aligned}
d\left(z, x_{n}\right) & \leq \rho+\frac{z}{z} \cdot \rho_{M}(x, y, z, \rho, \rho+k)-\rho_{M}(x, y, z, \rho, \rho+k) \\
& <\rho,
\end{aligned}
$$

contradicting the definition of asymptotic radius.

2.6. It follows from 2.5 that:

If $M$ is regularly locally uniformly convex, if $\left(x_{n}\right)$ is a bounded sequence in $M$ with asymptotic centre $c$, and if $z \in M$ and a real sequence $\left(\delta_{n}\right)$ and a positive integer $k$ are such that $\lim _{n \rightarrow \infty} \delta_{n}=0$ and for all $n$,

$$
d\left(x_{n+k}, z\right) \leq d\left(x_{n}, c\right)+\delta_{n},
$$

then $z=c$.

2.7. Hence we have the following extension of a fixed point theorem of Edelstein [3], though the work of Section 4 is needed to prove that it is such an extension.

If $M$ is a regularly locally uniformly convex metric space, if $f: M \rightarrow M$, and $x \in M$ are such that $\left(f^{n} x\right)$ has asymptotic centre $c$, and if there is a positive integer $k$ and a real sequence $\left(\delta_{n}\right)$ such that $\lim _{n \rightarrow \infty} \delta_{n}=0$ and for all but finitely mony $n$,

$$
d\left(f^{n+k} x, f c\right) \leq d\left(f^{n} x, c\right)+\delta_{n},
$$


then $c$ is a fixed point of $f$.

\section{Existence of Chebychev and asymptotic centres}

To obtain existence results the notion of uniform rotundity is further strengthened. The notation of the first section is again retained.

3.1. A set $N \subseteq M$ is called uniformly rotund in $M$ if, in the notation of 2.1, for all bounded subsets $B$ of $M$ and all real $\varepsilon, p, q>0, p \leq q$, there is $z \in M$ such that $\gamma_{N}(\varepsilon, B, z, p, q)=\inf \left\{\delta \delta_{N}(x, y, z, \delta): x, y \in B, d(x, y) \geq \varepsilon, p \leq \delta \leq q\right\}$ is positive.

In particular, if $M$ is uniformly rotund in $M$, then $M$ is called uniformly rotund.

3.2. If $M$ is complete then every nonempty, bounded $S \subseteq M$ which is uniformly rotund in $M$ has a Chebychev centre in $M$.

Proof. If the Chebychev radius $\rho$ of $S$ in $M$ is zero, then the result is trivial, so suppose that $\rho>0$. There is a sequence $\left(p_{n}\right)$ of points of $M$ such that for all $n$,

$$
S \subseteq B\left(p_{n}, \rho+1 / n\right)
$$

We prove that $\left(p_{n}\right)$ is a Cauchy sequence; it then follows that it has a limit which is a Chebycher centre of $S$ in $M$.

Suppose on the contrary that $\left(p_{n}\right)$ is not a Cauchy sequence; then for some $\varepsilon>0$, for infinitely many positive integers $n$, and for each such $n$ some $m \geq n, d\left(p_{m}, p_{n}\right) \geq \varepsilon$. Thus there is $z \in M$ such that for all such $m$ and $n$,

$$
\begin{aligned}
S & \subseteq B\left(p_{m}, \rho+1 / n\right) \cap B\left(p_{n}, \rho+1 / n\right) \\
& \subseteq B\left(z, \rho+1 / n-\gamma_{S}(\min (\varepsilon, 1), T, z, \rho, \rho+1)\right),
\end{aligned}
$$

where $T=B(S, \rho+1)$, which for $n$ sufficiently large contradicts the definition of $\rho$ as the Chebychev radius of $S$.

3.3. Similarly: 
Every bounded sequence $\left(x_{n}\right)$ in a complete, uniformly rotund metric space $M$ has an asymptotic centre.

3.4. It is immediate from 3.3 that if the hypotheses of 2.7 are strengthened by assuming that $M$ is nonempty, bounded, complete and uniformly rotund, then the hypothesis that $\left(f^{n} x\right)$ has an asymptotic centre can be omitted.

The following two fixed point theorems for nonexpansive mappings are examples of what can then be deduced. The former is the metric space version of the basic fixed point theorem for nonexpansive mappings; the latter has apparently not been mentioned previously.

(i) If $M$ is nonempty, bounded, complete and uniformly rotund, and if $f: M \rightarrow M$ is such that for all $x, y \in M$,

$$
d(f x, f y) \leq d(x, y),
$$

then $f$ has a fixed point.

(ii) If $M$ is as in (i), and if $f: M \rightarrow M$ is such that for alz $x, y \in M$,

$$
d(f x, f y) \leq \sum_{m=0}^{\infty} \alpha_{m}(x, y) \cdot d\left(x, f^{m} y\right),
$$

where $\left(\alpha_{m}(x, y)\right)$ is a sequence of nonnegative real numbers such that

$$
\sum_{m=0}^{\infty} \alpha_{m}(x, y) \leq 1,
$$

then $f$ has a fixed point.

Proof of (ii). Fix $x \in M$. The sequence $\left(f^{n} x\right)$ has an asymptotic centre $c$; we show that $c$ satisfies the conditions of 2.7 with $k=1$ and $\delta_{n}=0$.

Write $\rho$ for the asymptotic radius of $\left(f^{n} x\right)$; then for all $\varepsilon>0$, 


$$
\begin{aligned}
d\left(f c, f^{n+1} x\right) & \leq \sum_{m=0}^{\infty} \alpha_{m}\left(c, f^{n} x\right) \cdot d\left(c, f^{n+m} x\right) \\
& \leq \sum_{m=0}^{\infty} \alpha_{m}\left(c, f^{n} x\right) \cdot(\rho+\varepsilon) \text { for all but finitely many } n, \\
& \leq \rho+\varepsilon .
\end{aligned}
$$

\section{Connections with uniform convexity of Banach spaces}

4.1. We shall continue to use the term "uniform rotundity" as defined in 3.1; it should not be confused with the well known notion of uniform convexity of normed linear spaces, which may be defined as follows and has been called uniform rotundity by some authors.

4.2. A normed linear space $V$ is called uniformly convex if for all real $\varepsilon$ such that $0<\varepsilon<2$,

$$
\sup \left\{\frac{1}{2} .\|x+y\|: x, y \in V,\|x\| \leq 1,\|y\| \leq 1,\|x-y\| \geq \varepsilon\right\}>1 .
$$

We write $\delta(\varepsilon)$ for the difference between 1 and this supremum. We note first that:

4.3. If $V$ is a zoniformly convex normed linear space, if $T \subseteq V$ is convex and if $S \subseteq T$, then $S$ is uniformly rotund in $T$.

Proof. Fix real positive $\varepsilon, p$, and $q$, with $p \leq q$, and a bounded set $B \subseteq T$. Consider real $D$ such that $p \leq D \leq q, x, y \in B$ such that $\|x-y\| \geq \varepsilon$, and $w \in S \cap B(x, D) \cap B(y, D)$. As $T$ is convex, $\frac{3}{2} .(x+y) \in T$, so in the notation of 4.2 ,

$$
\begin{aligned}
D-\left\|\frac{1}{2} \cdot(x+y)-w\right\| & =D\left(1-\frac{1}{2} \cdot\left\|\frac{x-w}{D}+\frac{y-w}{D}\right\|\right) \\
& \geq D \cdot \delta(\varepsilon / D) \\
& \geq p \cdot \delta(\varepsilon / q) .
\end{aligned}
$$

4.4. It follows from 4.3 and 3.3 that 2.7 is indeed an extension of the fixed point theorem of Edelstein [3, Section 3, Theorem 1].

Consider now the converse of 4.3. For that purpose we mention the following lemma.

4.5. If $M$ is a metric space and $S \subseteq M$ is uniformly rotund in $M$, then the completion $\bar{S}$ of $S$ is uniformly rotund in the completion $\bar{M}$ of $M$. 
The following is a partial converse of 4.3 .

4.6. If $V$ is a normed linear space which is uniformly rotund in the sense of 3.1, then $V$ is uniformly convex in the sense of 4.2.

Proof. From 4.5 and 4.2 we may as well suppose that $V$ is a Banach space. To prove uniform convexity, fix $\varepsilon>0$. The hypotheses ensure that there is real $r<1$ such that for all $x, y \in B(0,1)$ such that $\|x-y\| \geq \varepsilon$, there is $z \in V$ such that

$$
I=B(x, I) \cap B(y, I) \subseteq B(z, r) \text {. }
$$

An elementary symmetry argument shows that $\frac{3}{2} \cdot(x+y)$ is the (unique) Chebychev centre of $I$, so for some $\rho \leq r$,

$$
I \subseteq B\left(\frac{1}{2} \cdot(x+y), \rho\right) \text {. }
$$

As in particular, $0 \in I$, we have $\left\|\frac{7}{2} \cdot(x+y)\right\| \leq r$, as required.

4.7. On the other hand there can be no complete converse of 4.3 , because:

Real Hilbert spaces (of dimension $\geq 2$ ) have subsets which are uniformly rotund but:not convex.

Proof. Fix a linear functional $f$ of unit norm on such a real Hilbert space $H$, and fix a real number $e$ such that

$$
\sqrt{15} / 4<e<1 \text {. }
$$

The following subset $S$ of $H$ will be shown to be uniformly rotund, though it is not convex:

$$
S=\{x \in H:\|x\|=1 \text { and } f x \geq e\} .
$$

To that end, fix real positive $p, q, \varepsilon$ such that $p \leq q$, and consider arbitrary $x, y \in S$ such that $\|x-y\|=\varepsilon$, real $D$ such that $p \leq D \leq q$, and

$$
w \in S \cap B(x, D) \cap B(y, D)
$$

We show that

$$
w \in B\left(\frac{x+y}{\|x+y\|}, D-\varepsilon^{2} \cdot k / 4\right)
$$

where $k>0$ is to be defined below.

Note that since there are such $x, y$, and $w, \varepsilon<2$ and $D \geq \varepsilon / 2$. 
By plane geometry in the plane of $x, y$, and $w$, writing $M$ for the diameter of $S$,

$$
\left\|w-\frac{1}{2}(x+y)\right\|^{2} \leq M^{2}-(\varepsilon / 2)^{2} .
$$

Likewise, working in the plane of $x, y$, and 0 ,

$$
\left\|\frac{1}{2} \cdot(x+y)-\frac{(x+y)}{\|x+y\|}\right\| \leq 1-\left(1-(\varepsilon / 2)^{2}\right)^{\frac{1}{2}} .
$$

Hence

$$
\begin{aligned}
\left\|w-\frac{x+y}{\|x+y\|}\right\| & \leq\left\|w-\frac{1}{2} \cdot(x+y)\right\|+\left\|\frac{1}{2} \cdot(x+y)-\frac{x+y}{\|x+y\| \|}\right\| \\
& \leq M-\varepsilon^{2} / 4 \cdot\left(\frac{1}{2} M-1\right) .
\end{aligned}
$$

The required inequality then follows from the fact that $M \leq 2\left(1-e^{2}\right)^{\frac{1}{2}}$, which implies that

$$
k=\frac{7}{2} M-1 \geq \frac{1}{4\left(1-e^{2}\right)^{\frac{1}{2}}}-1>0 .
$$

To see that $M \leq 2\left(1-e^{2}\right)^{\frac{1}{2}}$, suppose on the contrary that there are $a, b \in S$ such that $\|a-b\|>2\left(1-e^{2}\right)^{\frac{1}{2}}$, and deduce by elementary geometry in the plane of $a, b$, and 0 that $\left\|\frac{z}{2}(a+b)\right\|<e$; hence $f\left(\frac{a+b}{\|a+b\|}\right)>1$, a contradiction.

\section{Extensions of fixed point theorems of Cooper and Michael}

5.1. The following two fixed point theorems extend theorems of Cooper and Michael [1, Theorems 2.5 and 4.1], by removing all linearity assumptions. The first of these results also extends the corresponding result of Cooper and Michael in another way, which proves essential to the proof of the second result.

5.2 (i). If $X$ is a topological space with lower semicontinuous metric $d$, if $C \subseteq X$ is locally uniformly rotund and bounded with respect to $d$, and compact with respect to the topology, and if $D \subseteq C$ is nonempty, then all nonexpansive maps $T: C+C$ which map $D$ onto a dense subset of $D$ have a common fixed point.

In the above assertion, lower semicontinuity of $d$ means that for all 
$x, y \in X$ and all $\varepsilon>0$, there is an open set $U$ such that $x \in U$ and for all $z \in U, d(z, y) \geq d(x, y)-\varepsilon$.

(ii) If $X, C$ are as in (i) and if $H$ is a semigroup of continuous (with respect to the topology on $X$ ) nonexpansive mappings of $C$ into $C$, which is left reversible, then all the mappings of $H$ have a common fixed point.

In this assertion, left reversibility of $H$ means that for all $T, T^{\prime} \in H$,

$$
T H \cap T^{\prime} H \text { is not empty. }
$$

5.3. The proofs of these results given below are similar in structure to the corresponding proofs of Cooper and Michael; we emphasize only the differences.

5.4. Throughout the rest of this section $X, d, C, D$, and $H$ are as in 5.2. We also write $T$ for the topology on $X, \gamma_{C}(D)$ for the set of Chebychev centres of $D$ in $C$, and for each $x \in X$, we define $r_{D}(x)$ by:

$$
r_{D}(x)=\sup _{y \in D} d(x, y)
$$

5.5. $r_{D}$ is Zower semicontinuous (with respect to $T$ ) on $C$.

That is, for all $x \in C$ and $\varepsilon>0$, there is a T-open neighbourhood $U$ of $x$ such that for all $y \in U$,

$$
r_{D}(y) \geq r_{D}(x)-\varepsilon \text {. }
$$

5.6. $\gamma_{C}(D)$ is nonempty, compact (with respect to $T$ ) and bounded (with respect to $d$ ).

The proofs of both 5.5 and 5.6 are substantially the same as the corresponding proofs of Cooper and Michael, so we omit them.

5.7. Every nonexpansive mapping $T: C \rightarrow C$ which maps $D$ onto a dense subset of $D$, maps $\gamma_{C}(D)$ into $\gamma_{C}(D)$.

Proof. Given $x \in \gamma_{C}(D)$, show as follows that $T x \in \gamma_{C}(D)$. For arbitrary $y \in D$, write $\left(T y_{n}\right)$ for a sequence of points convergent to 
$y$, where $y_{n} \in D$ for all $n$. Then for all $n$,

$$
\begin{aligned}
d(T x, y) & \leq d\left(T x, T y_{n}\right)+d\left(T y_{n}, y\right) \\
& \leq d\left(x, y_{n}\right)+d\left(T y_{n}, y\right) \\
& \leq \rho+d\left(T y_{n}, y\right),
\end{aligned}
$$

where $\rho$ is the Chebychev radius of $D$. Hence $d(T x, y) \leq \rho$, as required.

5.8. If in particular, $C$ is locally uniformly rotund, then $\gamma_{C}(D)$ consists of a single point $(2.2,2.3)$, so we have the result 5.2 (i).

5.9. Consider now the proof of 5.2 (ii). By the usual Zorn's Lemma argument (as in Cooper and Michael) there is a minimal subset $K_{0}$ of $C$ which is nonempty, compact with respect to $T$ and such that $T K_{0} \subseteq K_{0}$ for all $T \in H$. We show that for all $T \in H, T K_{0}=K_{0}$. The result

5.2 (ii) then follows from 5.2 (i) when one takes $D$ to be $K_{0}$; thus this argument depends on allowing $D$ to be a proper subset of $C$ - a case not covered by Cooper and Michael's result.

To prove $T K_{0}=K_{0}$, define $K_{0}^{\prime}=\bigcap_{T \in H} T K_{0}$ and use the leftreversibility of $H$ (as in Cooper and Michael) and the continuity of the maps in $H$ to show that $K_{0}^{\prime}$ is nonempty, compact and invariant under all $T \in H_{0}$. Thus, by the minimality of $K_{0}$ in this respect, $K_{0}^{\prime}=K_{0}$, so $T K_{0}=K_{0}$ for all $T \in H$, as required.

\section{References}

[1] T.J. Cooper and J.H. Michael, "Two fixed point theorems and invariant integrals", Bull. Austral. Math. Soc. 11 (1974), 15-30.

[2] Michael Edelstein, "The construction of an asymptotic centre with a fixed point property", Bull. Amer. Math. Soc. 78 (1972), 206-208. 
[3] Michael Edelstein, "Fixed point theorems in uniformly convex Banach spaces", Proc. Amer. Math. Soc. 44 (1974), 369-374.

Department of Mathematics,

Queensland Institute of Technology, North Quay,

Queensland. 